\section{Latent Impurities in Electrodes used for Spectrographic Research}

IT has often been pointed out that for accurate results in quantitative spectrographic analysis, the standards of known composition, against whose spectra that of the specimen to be analysed is matched, should be made up on a base that simulates as closely as possible the composition of the specimen. This necessity arises from the fact that the intensity of the spectrum shown by a minor constituent of a mixture depends not only on the concentration of the element in question, but also on the nature of the in the 'blank' spectrum, may leap into prominence as soon as any mixture that approximately resembles a tissue ash is introduced into the arc.

The accompanying illustrations (Figs. 1 and 2) show the effect of introducing pure sodium chloride into the arc struck between graphite electrodes, in which the principal impurities are vanadium, calcium and iron. ('The manufacturers' claim that the sodium chloride is spectroscopically pure, except for a trace of silver, was verified by examining its spectrum in the silver are.) It is clear that those lines of vanadium, calcium, iron and copper which are only with some difficulty visible in the spectrum given by the electrodes alone are greatly intensified by the presence of sodium atoms in the arc, while several lines of titanium, which could not be detected at all in the spectrum of the electrodes, also become visible on the plate, though they are not strong enough to show up in the reproduction. The chemical report accompany. ing the olectrodes states that they contain 0.2 parts of titanium per million. The lines of silicon and magnesium, on the other hand, show no sign of intensification.

Iig. 1.

$\begin{array}{lc}\mid \mathrm{Cu} & \mathrm{Na} \\ \mathrm{Cu} & \mathrm{Na} \\ 3247 & 3302 \\ 327 \pm & 3303\end{array}$

3184 $3185 \cdot 4$ major constituents. It has been shown, for example, that in the case of dilute solutions of lead salts, the lead lines are much stronger if the solution contains a considerable amount of zinc ${ }^{1}$.

In the course of some analyses of animal tissues, which were begun in the Physics Dopartmont, Trinity College, Dublin, and are being continued in Cambridge, it has become obvious that this consideration applies not only to the constituents of the specimen, but also to impurities in the electrodes, and that failure to take account of it may render qualitative as well as quantitative results unreliable. The

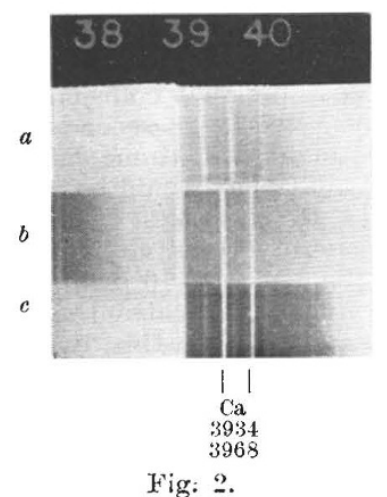

majority of workers in this field, and particularly those who were seeking only qualitative results, and did not, in consequence, have occasion to compare their specimens with standards of known composition, appear to have followed the practice recommended in all the practical handbooks of spectroscopy, of regarding a 'blank' spectrum of the electrodes alone, photographed in juxtaposition to the spectrum of the specimen, as being a sufficient safeguard against interpreting impurities in the electrodes as constituents of the specimen. But the fact is that in the case of carbon or graphite electrodes, impurities the strongest lines of which can only be faintly discerned
These results are in harmony with some observations of Papish and O'Leary ${ }^{2}$, who found, using graphite electrodes, that the amount of chromium required to produce a spectrum of standard intensity was about sixteen times as great when a dilute solution of chromic acid was put on the electrodes as when an artificial ruby was used. Nobody appears, however, to have drawn from this observation the moral concerning the behaviour of impurities. It is hoped shortly to publish elsewhere, together with the results of the analyses themselves, a list of the elements that are likely, on account of this effect, to give misleading results in biological analyses. Meanwhile, it is clear that reports, by workers who have used carbon or graphite electrodes, on the distribution in living tissues of vanadium, titanium, copper, and possibly other elements, must be treated with reserve.

\section{University Department of Zoology, Cambridge.}

Dec. 31 .

${ }_{1}^{1}$ Nitchie, C. (., Ind. and Eng. Chem. (Anal. Ed.), 1, 1 (1929).

'Papish, J., and O'Leary, W. J., Ind. and Eng. Chem., 3, 11 (1931).

\section{Luminescence of Solutions of Terbium Salts}

VERY dilute aqueous solutions of terbium salts exhibit a bright luminescence when exeited by the ultra-violet rays ${ }^{1}$. 'The maximal excitation is caused by the absorption of the wave-lengths coinciding with the broad absorption band of terbium, stretch. ing from $290 \mathrm{~m} \mu$ far towards the short wave-lengths ${ }^{2}$. The spectrurn of this luminescence consists of 7 bands, each band being nearly $100 \mathrm{~A}$. wide. The wavelengths and frequencies of the middles of the bands are given in the accompanying table.

The second column gives a rough estimation of the intensities of the bands. It is seen that all the differences between the frequencies of the bands are fairly well expressed by the equation :

$$
\Delta v_{k}=K \Delta v
$$

